\title{
Juvenile arthritis caused by a novel FAMIN (LACC1) mutation in two children with systemic and extended oligoarticular course
}

\author{
Tilmann Kallinich ${ }^{1,2^{*}}$ D Anne Thorwarth ${ }^{1,2+}$, Sae-Lim von Stuckrad ${ }^{1,2}$, Angela Rösen-Wolff ${ }^{3}$, Hella Luksch ${ }^{3}$, \\ Patrick Hundsdoerfer ${ }^{4}$, Kirsten Minden ${ }^{1,2,5}$ and Peter Krawitz ${ }^{6}$
}

\begin{abstract}
Background: The pathophysiological origin of juvenile idiopathic arthritis (JIA) is largely unknown. However, individuals with presumably pathogenic mutations in FAMIN have been reported, associating this gene with a rare subtype of this disorder. FAMIN, that is formerly also referred to as LACC1 or C13orf31, has recently been shown to play a crucial role in immune-metabolic functions and is involved in regulation of inflammasome activation and promotion of ROS production.

Case presentation: We describe two siblings with severe familial forms of juvenile arthritis in which whole-exomesequencing revealed a novel homozygous frameshift mutation (NM_153218.2:C.827delC. p.(T276fs*2) in FAMIN.

Conclusions: The observation of a new deleterious mutation adds further evidence that pathogenic mutations in FAMIN are causal for a monogenic form of JIA. Furthermore the associated phenotype is not restricted to systemic $J I A$, but can also be found in other forms of familial juvenile arthritis.
\end{abstract}

Keywords: Systemic juvenile idiopathic arthritis, Exome sequencing, FAMIN, LACC1

\section{Background}

Despite intensive analyses, the precise mechanisms leading to the phenotype of systemic juvenile idiopathic arthritis (sJIA) are still unknown (reviewed by [1]). Current understanding favors a model in which deregulation of innate immune mechanisms causes an upregulation of various soluble mediators, e.g. IL-1 $\beta$ [2], IL-18 [3], IL-6 [4] and the S100 molecules [5] and orchestrates a proinflammatory response reaction. Furthermore, an association of polymorphisms within the ILI and IL1R cluster genes [6,7], the macrophages migration inhibitor factor (MIF) and the IL10 cytokine cluster [8] has previously been described. Furthermore, a current genome wide association study (GWAS) identified the MHC

\footnotetext{
* Correspondence: tilmann.kallinich@charite.de

${ }^{\dagger}$ Equal contributors

${ }^{1}$ Charité University Medicine Berlin, Pediatric Pneumology and Immunology,

Augustenburger Platz 1, 13353 Berlin, Germany

${ }^{2}$ Center for Chronically Sick Children of the Charité, Augustenburger Platz 1,

13353 Berlin, Germany

Full list of author information is available at the end of the article
}

locus as a bona fide susceptibility locus for the phenotype of sJIA [9].

In a previous report familial cases with sJIA have been linked to the gene FAMIN, ('fatty acid metabolism - immunity nexus', formerly also referred to as LACC1 or C13orf31). Wakil et al. identified the homozygous missense mutation, p.Cys284Arg, in affected individuals from consanguineous Saudi Arabian families [10]. Furthermore, Arostegui, et al. reported a frame-shift mutation, p.Cys43Tyrfs*6, in three siblings from a consanguineous Moroccan family with severe highly inflammatory rheumatoid factor-negative polyarticular JIA [11].

These clinical reports coincide with a very recently published study that describe FAMIN as central regulator of endogenous fatty acid synthesis and their mitochondrial oxidation in macrophages [12]. In this respect, intact FAMIN is crucial in the production of inflammasome-mediated IL- $1 \beta$ as well as reactive

(c) The Author(s). 2016 Open Access This article is distributed under the terms of the Creative Commons Attribution 4.0 International License (http://creativecommons.org/licenses/by/4.0/), which permits unrestricted use, distribution, and reproduction in any medium, provided you give appropriate credit to the original author(s) and the source, provide a link to the Creative Commons license, and indicate if changes were made. The Creative Commons Public Domain Dedication waiver (http://creativecommons.org/publicdomain/zero/1.0/) applies to the data made available in this article, unless otherwise stated. 
oxygen species (ROS). FAMIN mutations are primarily described as having a loss-of-function effect on metabolic cell function and subsequent immunological response. Nevertheless, high dosages of lipopolysaccharides (LPS) induced catastrophic activation of IL-1 $\beta$ in ${ }_{F A M I N}{ }^{-1-}$ mice, indicating that the profoundly impaired 'energetic reserves' might predipose these cells towards a pyroptotic, pro-death response.

We here present two siblings with severe forms of juvenile arthritis both harbouring a novel homozygous frameshift mutation within FAMIN. One sibling additionally developed precursor-B cell acute lymphoblastic leukemia (ALL). This report extends the phenotype of monogenic juvenile arthritis and adds evidence for the causative role of FAMIN in these cases.

\section{Case presentation}

The consanguineous family originates from Lebanon (Fig. 1). The two affected girls as well as the two healthy siblings were born in Germany. Neither the parents nor the other siblings ever showed evidence of arthritis, systemic inflammation or recurrent infections.

At the age of 16 months the older child (II-3) developed simultaneously severe polyarthritis of wrists, knees and ankles, lymphadenopathy, extreme sensitivity to body contact, quotidian fever and remarkably elevated inflammatory markers (CRP $121 \mathrm{mg} / \mathrm{l}$, leukocytes 18/nl). No serositis or exanthema was noticed. After intensive exclusion of other causes including bone marrow aspiration, systemic juvenile idiopathic arthritis was diagnosed according to the International League of Associations for Rheumatology (ILAR)-criteria.

The treatment consisted of repeated intraarticular steroid injections (shoulders, elbows, hands, hips, knees and ankles), non-steroidal anti-inflammatory drugs, high doses of corticosteroids $(2 \mathrm{mg} / \mathrm{kg})$ and methotrexate. Etanercept and Anakinra did not lead to a sufficient

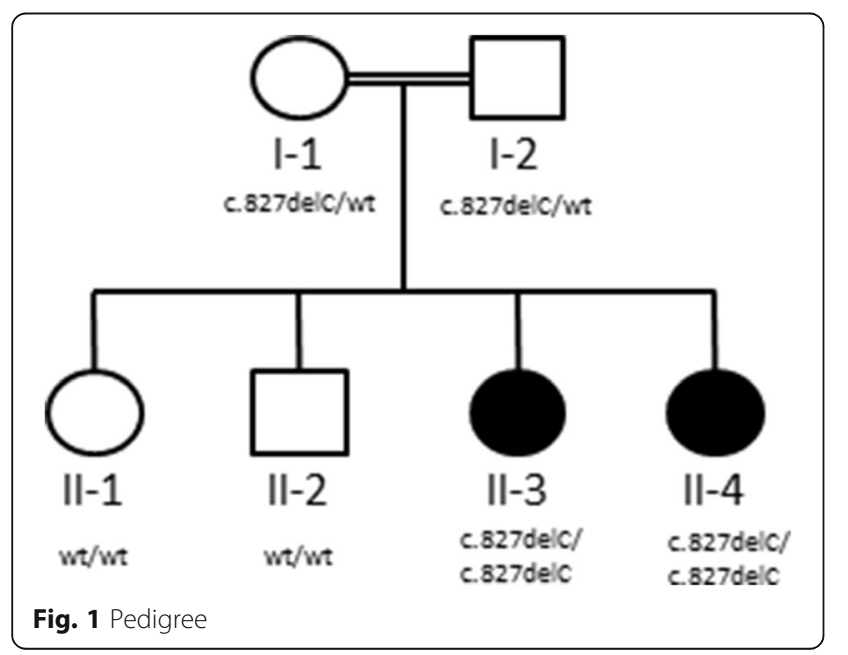

disease control. Finally, clinical remission was achieved 3,5 years after introduction of tocilizumab and methotrexate in addition to low dose steroids $(0,2 \mathrm{mg} / \mathrm{kg})$ and non-steroidal anti-inflammatory drugs.

At the age of six, the girl developed thrombocytopenia (56/nl) and precursor-B cell ALL was diagnosed. Over seven months the girl was treated with intensive chemotherapy according to the AIEOP-BFM ALL 2009 protocol. During this time she repeatedly suffered from severe flares of arthritis even shortly after chemotherapy (e.g. cyclophosphamide $1 \mathrm{~g} / \mathrm{m}^{2}$ ), which resolved spontaneously.

At the age of eight, ALL relapse (bone marrow and central nervous system) was diagnosed. Second remission was achieved by chemotherapy according to the ALL-REZ BFM 2002 protocol. Allogeneic bone marrow transplantation from an unrelated 9/10 HLA-identical donor was performed after total body irradiation (12Gy + 6 Gy CNS boost) and high-dose chemotherapy. Posttransplant course was complicated by acute grade IV graft-versus-host disease of liver and skin. With respect to the ALL and the juvenile arthritis the girl is in continuous remission without immunosuppressive therapy since 24 months after bone marrow transplantation.

The seven-year-old female sister of the same family (II- 3) developed first manifestations of arthritis (knee and ankles) at the age of 15 months, which was initially classified as oligoarticular juvenile idiopathic arthritis and treated with intraarticular steroidinstillation and nonsteroidal anti-inflammatory drugs.

Eight months later the juvenile idiopathic arthritis flared up with a symmetric polyarthritis (shoulder, elbows, knees, ankles, wrists) and thus reclassified as extended oligoarticular JIA. Unusually high inflammatory makers (e.g. CRP $221 \mathrm{mg} / \mathrm{l}$, leucocytes 18/nl) were noticed, but fever, rash, organomegaly or lymphadenopathy was not present. Treatment with methotrexate and systemic steroids $(1 \mathrm{mg} / \mathrm{kg})$ was started. Due to uncontrolled systemic inflammation adalimumab in combination with hydroxychloroquine was introduced and sufficient control of the inflammation was achieved.

In both children whole-exome-sequencing revealed the homozygous one base pair deletion in FAMIN NM_153218.2:c.827delC. The mutation results in a frameshift and a premature stop codon, p.(Thr276fs"2). Both parents are heterozygous carriers of the deletion and the two unaffected children showed the wildtype of the gene.

\section{Conclusion}

In this report we describe the novel homozygous frameshift mutation, p.Thr276fs 2 , in FAMIN. The observation that a frameshift mutation leads to a similar juvenile arthritis phenotype comparable to the previously described 
mutations strongly supports the significance of these gene variants in the development of juvenile arthritis. Our cases demonstrate, that FAMIN mutations can also be found in extended oligoarticular juvenile arthritis associated with systemic inflammation.

Interestingly, all case reports with deleterious mutations in FAMIN have so far been from families of Arab ethnicity. However, this might simply be due to the fact, that the identification of pathogenic mutations is facilitated in highly consanguineous families. It is noteworthy, that none of the reported pathogenic mutations has been observed in the ExAC cohort.

Previously, genome wide association studies (GWAS) of unrelated patients with sIIA did not reveal significant $-\log _{10} \mathrm{p}$ values for the FAMIN locus [9]. Therefore, variants within this gene do not seem to play a role in patients suffering from the common forms of sJIA. However, GWA studies can only detect frequent variants. Cases of juvenile arthritis caused by FAMIN mutations have to be distinguished from the common forms of (s)JIA. Since these cases are not restricted to systemic forms and are not 'idiopathic' they should be referred to as monogenic juvenile arthritis. The severity of the phenotypes in terms of difficult disease control underlines the clinical need to separate these entities from common forms of sJIA. Whether the phenotype of monogenic juvenile arthritis is restricted to familial cases has to be analyzed in larger cohort studies.

Large GWAS, including several thousands of patients with infectious and autoimmune diseases give evidence to a major role of FAMIN in the inflammatory response: in patients with leprosy an association of the common variant p.Ile254Val (rs37641479) located within FAMIN was found and the gene activity is discussed as a hostresistance factor in leprosy [13-16]. The same variant was found in cohorts with increased susceptibility to Crohn's disease pointing to a common host response pathway in leprosy and Crohn's disease $[17,18]$. Furthermore, the 'familial sJIA variant' p.Cys284Arg was detected in a family with early-onset Crohn's disease [19]. Crohn's disease and infections share some phenotypical aspects of JIA, e.g. the occurrence of arthritis and the systemic inflammatory reaction. Current pathophysiological concepts of JIA furthermore indicate an important role of a dysregulation within pathogen-sensing mechanisms, e.g. the inflammasomes. Together with the observation of similar genetic alterations this suggests that pathophysiological pathways are shared in these three diseases. Moreover, the now known function of FAMIN and the described variants causing different forms of JIA these underlines that the found variants are not simply in tight linkage disequilibrium with a causative variant but are truly causative.
When introduced into a murine model, the variants p.Cys284Arg, associated with 'familial sJIA' and early onset Crohn's disease, as well as the SNP p.Ile254Val associated with increased susceptibility to Crohn's disease and leprosy, suppressed the observed metabolic changes as measured by means of extracellular acidification rate and oxygen-consumption rate [12]. Thus, an ex vivo assay exists to determine the gene-dose effect on metabolic cell function.

The altered metabolic cell function observed ex vivo, reflects the severity of clinical phenotypes. In this respect, p.Ile254Val led to rather mild cellular changes with increased susceptibility to leprosy and Crohn's disease, whereas the p.Cys284Arg variant caused stronger metabolic alterations resulting in a more severe phenotype with early-onset Crohn's disease or 'familial sJIA', respectively. This genotype-phenotype correlation is furthermore supported as the novel frameshift mutation p.Thr276fs reported here is of higher predicted pathogenicity than the missense variant p.Cys284Arg. This hypothesis is supported by the fact, that the stop codon at amino acid position 276 is located upstream of 284 and therefore affects the consecutive $\mathrm{N}$-terminal amino acids 288 to 430.

After BMT, the child (II-3) does not show any signs of arthritis. Given the fact, that FAMIN is closely linked to the inflammatory function of macrophages, this observation underlines the pathophysiological importance of this cell type in sJIA.

Taken together, we present a family with closely related parents with two children suffering of severe forms of monogenic juvenile arthritis caused by homozygous variants within FAMIN. In line with the previously described cases of FAMIN-related familial systemic and polyarticular JIA, the reported association with other inflammatory and infectious diseases, respectively, as well as the currently published pathophysiological role of FAMIN, these cases add evidence for a causative role of the alterations in the development of these inflammatory phenotypes.

\section{Abbreviations \\ BMT: Bone marrow transplantation; GWAS: Genome wide association study; JIA: Juvenile idiopathic arthritis; LPS: Lipopolysaccharides; MIF: Migration inhibitor factor; ROS: Reactive oxygen species; sJIA: Systemic juvenile idiopathic arthritis}

\section{Acknowledgements}

We thank the patients and their family.

\section{Funding}

This work was supported by a grant of "Deutsche Forschungsgemeinschaft" to PK (DFG KR 3985/6-1).

\section{Availability of data and materials}

The datasets generated during and/or analysed during the current study are available from the corresponding author on reasonable request. 


\section{Authors' contributions}

TK, S-L v S, PH and KM are the care giving physicians; TK, AT, A R-W, HL and PK were responsible for study conception and design as well as data acquisition, all authors were involved in manuscript drafting and revising it critically for intellectual content and approved the final version.

\section{Competing interests}

The authors declare that they have no competing interests.

\section{Consent for publication}

Written consent for publication was obtained from the parents.

\section{Ethics approval and consent to participate}

Publication was approved by ethical committee Charite University Medicine Berlin (EA2/108/16).

\section{Author details}

'Charité University Medicine Berlin, Pediatric Pneumology and Immunology, Augustenburger Platz 1, 13353 Berlin, Germany. ${ }^{2}$ Center for Chronically Sick Children of the Charité, Auqustenburger Platz 1, 13353 Berlin, Germany. ${ }^{3}$ Department of Pediatrics, University Clinic Carl Gustav Carus, TU Dresden, Fetscherstr. 74, 01037 Dresden, Germany. ${ }^{4}$ Charité University Medicine Berlin, Pediatric Oncology and Hematology, Augustenburger Platz 1, 13353 Berlin, Germany. ${ }^{5}$ Department of Rheumatology and Clinical Immunology, Charité University Medicine Berlin, Charitéplatz 1, 10117 Berlin, Germany. ${ }^{6}$ Charité University Medicine Berlin, Institute of Medical Genetics and Human Genetics, Augustenburger Platz 1, 13353 Berlin, Germany.

\section{Received: 19 September 2016 Accepted: 16 November 2016}

Published online: 24 November 2016

\section{References}

1. Bruck N, Schnabel A, Hedrich CM. Current understanding of the pathophysiology of systemic juvenile idiopathic arthritis (sJIA) and target-directed therapeutic approaches. Clin Immunol. 2015;159:72-83. doi:10.1016/j.clim.2015.04.018.

2. Pascual V, Allantaz F, Arce E, Punaro M, Banchereau J. Role of interleukin-1 (IL-1) in the pathogenesis of systemic onset juvenile idiopathic arthritis and clinical response to IL-1 blockade. J Exp Med. 2005;201:1479-86. doi:10.1084/jem.20050473.

3. de Jager W, et al. Blood and synovial fluid cytokine signatures in patients with juvenile idiopathic arthritis: a cross-sectional study. Ann Rheum Dis. 2007:66:589-98. doi:10.1136/ard.2006.061853.

4. Pignatti $P$, et al. Abnormal regulation of interleukin 6 in systemic juvenile idiopathic arthritis. J Rheumatol. 2001;28:1670-6.

5. Wittkowski $\mathrm{H}$, et al. S100A12 is a novel molecular marker differentiating systemic-onset juvenile idiopathic arthritis from other causes of fever of unknown origin. Arthritis Rheum. 2008;58:3924-31. doi:10.1002/art.24137.

6. Stock CJ, et al. Comprehensive association study of genetic variants in the IL-1 gene family in systemic juvenile idiopathic arthritis. Genes Immun. 2008:9:349-57. doi:10.1038/gene.2008.24.

7. Meazza C, et al. Macrophage migration inhibitory factor in patients with juvenile idiopathic arthritis. Arthritis Rheum. 2002;46:232-7. doi:10.1002/ 1529-0131(200201)46:1<232::AID-ART10059>3.0.CO;2-B.

8. Hedrich CM, Bream JH. Cell type-specific regulation of IL-10 expression in inflammation and disease. Immunol Res. 2010;47:185-206. doi:10.1007/s12026-009-8150-5.

9. Ombrello MJ, et al. HLA-DRB1*11 and variants of the MHC class II locus are strong risk factors for systemic juvenile idiopathic arthritis. Proc Natl Acad Sci U S A. 2015;112:15970-5. doi:10.1073/pnas.1520779112.

10. Wakil SM, et al. Association of a mutation in LACC1 with a monogenic form of systemic juvenile idiopathic arthritis. Arthritis Rheum. 2015;67:288-95. doi:10.1002/art.38877.

11. Arostegui II, et al. A family carrying a homozygous LACC1 truncated mutation expands the clinical phenotype of this disease beyond systemiconset juvenile idiopathic arthritis. Pediatr Rheumatol. 2015;13 Suppl 1:076.

12. Cader MZ, et al. C13orf31 (FAMIN) is a central regulator of immunometabolic function. Nat Immunol. 2016. doi:10.1038/ni.3532.

13. Zhang FR, et al. Genomewide association study of leprosy. N Engl J Med. 2009;361:2609-18. doi:10.1056/NEJMoa0903753.
14. Sales-Marques C, et al. NOD2 and CCDC122-LACC1 genes are associated with leprosy susceptibility in Brazilians. Hum Genet. 2014;133:1525-32. doi:10.1007/s00439-014-1502-9.

15. Grant AV, et al. Crohn's disease susceptibility genes are associated with leprosy in the Vietnamese population. J Infect Dis. 2012;206:1763-7. doi:10.1093/infdis/jis588.

16. Liu H, et al. Discovery of six new susceptibility loci and analysis of pleiotropic effects in leprosy. Nat Genet. 2015;47:267-71. doi:10.1038/ng.3212.

17. Franke A, et al. Genome-wide meta-analysis increases to 71 the number of confirmed Crohn's disease susceptibility loci. Nat Genet. 2010;42:1118-25. doi:10.1038/ng.717.

18. Jostins $L$, et al. Host-microbe interactions have shaped the genetic architecture of inflammatory bowel disease. Nature. 2012;491:119-24. doi:10.1038/nature11582.

19. Patel N, et al. Study of Mendelian forms of Crohn's disease in Saudi Arabia reveals novel risk loci and alleles. Gut. 2014;63:1831-2. doi:10.1136/gutjnl2014-307859.

\section{Submit your next manuscript to BioMed Central and we will help you at every step:}

- We accept pre-submission inquiries

- Our selector tool helps you to find the most relevant journal

- We provide round the clock customer support

- Convenient online submission

- Thorough peer review

- Inclusion in PubMed and all major indexing services

- Maximum visibility for your research

Submit your manuscript at www.biomedcentral.com/submit

) Biomed Central 